\title{
Moderation of the Flux Jumps Dynamics by Eddy-Currents in a Disk Shape NbTi Superconductor
}

\author{
S. Vasiliev ${ }^{a, *}$, V.V. Chabanenko ${ }^{b}$, A. Nabialek ${ }^{a}$, \\ V. Rusakov ${ }^{c}$, S. Piechota ${ }^{a}$ AND H. SzymczaK ${ }^{a}$ \\ ${ }^{a}$ Institute of Physics, Polish Academy of Sciences \\ al. Lotników 32/46, 02-668 Warsaw, Poland \\ ${ }^{b}$ Donetsk Physico-Technical Institute, Ukrainian Academy of Sciences \\ 72 R. Luxemburg str., 83114, Donetsk, Ukraine \\ ${ }^{c}$ Donetsk National University \\ Universitetskaya str. 24, 83-055 Donetsk, Ukraine
}

\begin{abstract}
We studied the moderation of the flux jumps dynamics in a disc shape $\mathrm{NbTi}-50 \%$ superconductor caused by eddy-currents induced in two copper cylinders attached to both surfaces of the investigated sample. We investigated experimentally the time of the flux jump duration, amount of the magnetic flux entering the sample during the jump as well as the fine structure of the jumps as a function of temperature and the external magnetic field. A simple theoretical model, which describes the magnetic field dependence of the amount of the magnetic flux entering the superconducting sample during the flux jump, was developed.
\end{abstract}

PACS numbers: 74.60.Ec, 74.60.Ge

\section{Introduction}

Above the first critical magnetic field, $H_{\mathrm{c} 1}$, magnetic flux enters into type II superconductors as quantized flux lines called Abrikosov vortices. The vortices motion depends on the presence of pinning centers, which lead to nonuniform flux distribution inside the superconductors. The penetrating field is localized near the surface of the sample. The gradient of magnetic induction in superconducting

*corresponding author; e-mail: vasil@ifpan.edu.pl 
sample $(\Delta B)$ corresponds to marginally stable critical state and under certain conditions the system can become unstable. At the constant external magnetic field, $H_{\text {ext }}$, depinning jumps of vortices (or of the stocks of vortices) occur under thermal fluctuations. If the external magnetic field increases, the gradient $\Delta B$ increases too. If the repulsion between vortices within the screening layer exceeds the pinning forces the flux front moves into the sample. This movement consists of a series of flux jumps of different scales [1-3]. In our studies we investigated global flux jumps, which take place at magnetothermal instability [4]. In this case the vortices of the entire volume of the sample are involved into the motion. In our previous work we investigated time dependence of local surface magnetization, or of local surface induction, during the magnetothermal instability using miniature Hall probes [5]. Our investigations have shown complicated dynamics of a local field on the surface of the superconductor.

In the present work we investigated an influence of the eddy-currents induced in a normal metal attached to the superconducting sample on the flux jumps dynamics. In particular, we studied temperature and magnetic field dependence of the time of the flux jump duration, the amount of the magnetic flux entering the sample during the jump as well as the fine structure of the jumps.

\section{Experimental details}

The magnetic flux dynamics of the NbTi-50\% disc shape sample (diameter $14 \mathrm{~mm}$, height $4 \mathrm{~mm}$ ) was studied by pick-up coils wound directly on sample and contained three turns of copper wire. The voltage from pick-up coils $E_{\text {coil }} \propto \mathrm{d} \Phi / \mathrm{d} t$ was registered by the transient recorder (TCC-1000, Riken Denshi Co., Ltd.) with

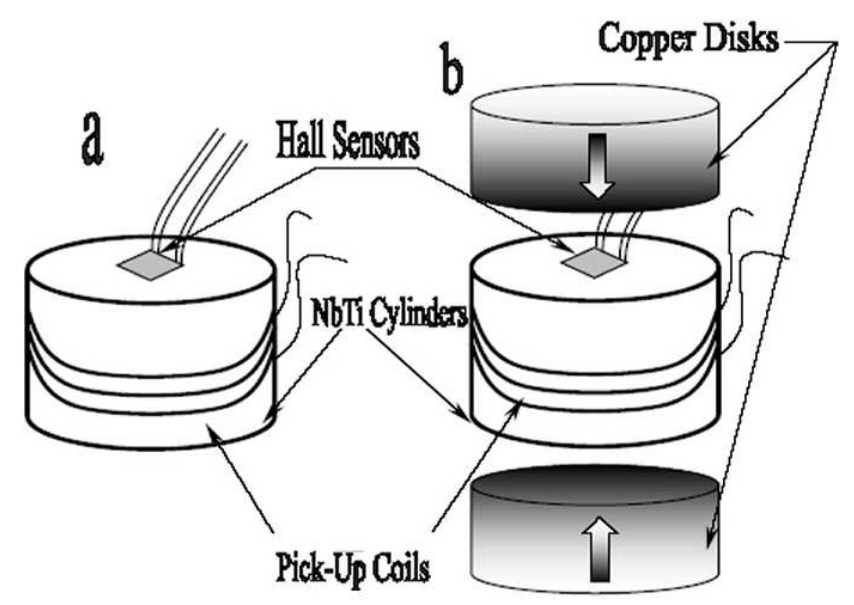

Fig. 1. Experimental geometry (a) superconducting disk, (b) superconducting disk with two copper disks. Hall sensors and pick-up coils are also shown. 
memory. Surface magnetization hysteresis loops were measured using two miniature Hall probe sensors. The first sensor chip ( $n$-InSb thin film layers doped with Sn) was placed in the center of the sample and measured surface induction, while the second one measured the induction of the external magnetic field. The sensitivity of the Hall probes was about $100 \mu \mathrm{V} / \mathrm{mT}$. A difference between the signals from both Hall sensors was proportional to a local surface magnetization (Fig. 1a). A thermocouple thermometer attached to the sample monitored its temperature. Our investigations were performed in a 12 tesla superconducting magnet with variable temperature insert. The external magnetic field was swept with the constant rate of about $1 \mathrm{~T} / \mathrm{min}$. An influence of the eddy-currents on the flux jumps dynamics was studied using two copper disks (diameter $14 \mathrm{~mm}$, height $4 \mathrm{~mm}$ ) attached to both surfaces of the superconducting disc (Fig. 1b).

\section{Results and discussion}

The hysteresis loops of surface magnetization, $M_{\text {surface }}(H)$, as well as the signal from the pick-up coil, which shows the structure of the jump, in the situation of a superconducting sample without copper discs are shown in Figs. 2 and 3 for the temperatures of $2 \mathrm{~K}$ and $4.2 \mathrm{~K}$, respectively. Figures 4 and 5 show the hysteresis loops of surface magnetization and the signal from the pick-up coils in a situation

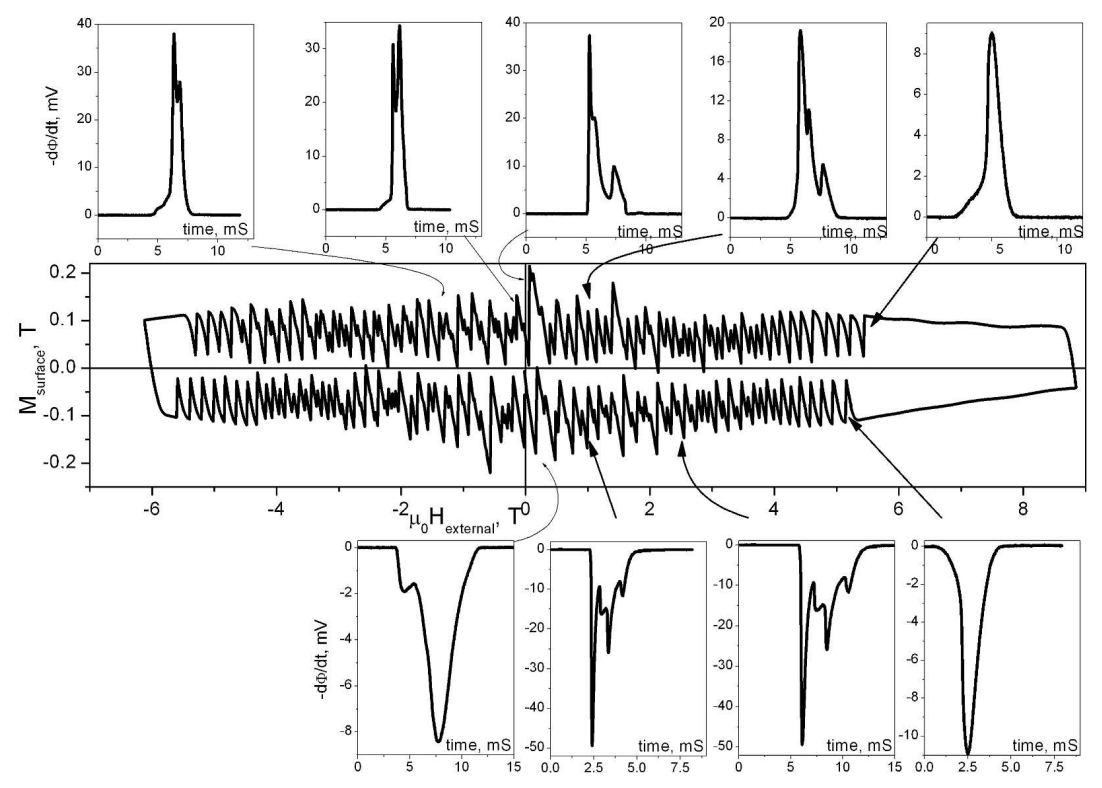

Fig. 2. Surface magnetization hysteresis loop (middle part) and the signals taken from the pick-up coil during the following flux jumps (upper and lower parts) at $2 \mathrm{~K}$. Superconducting sample without copper disks. 


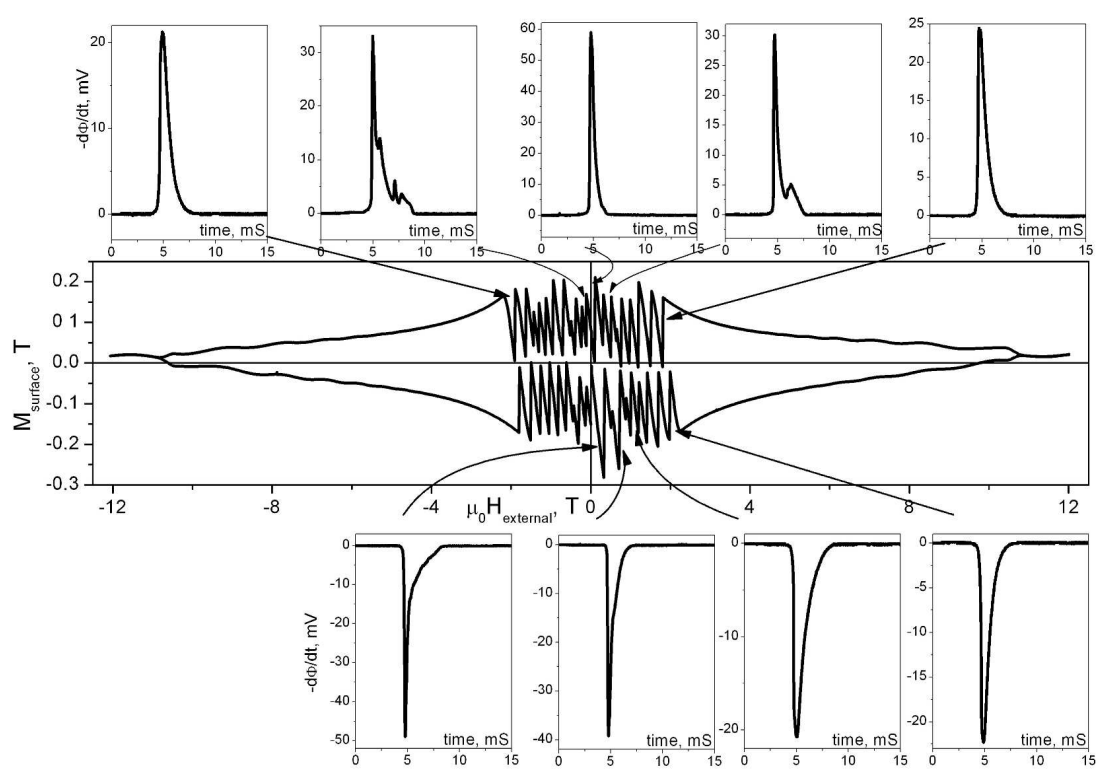

Fig. 3. Surface magnetization hysteresis loop (middle part) and the signals taken from the pick-up coil during the following flux jumps (upper and lower parts) at $4.2 \mathrm{~K}$. Superconducting sample without copper disks.

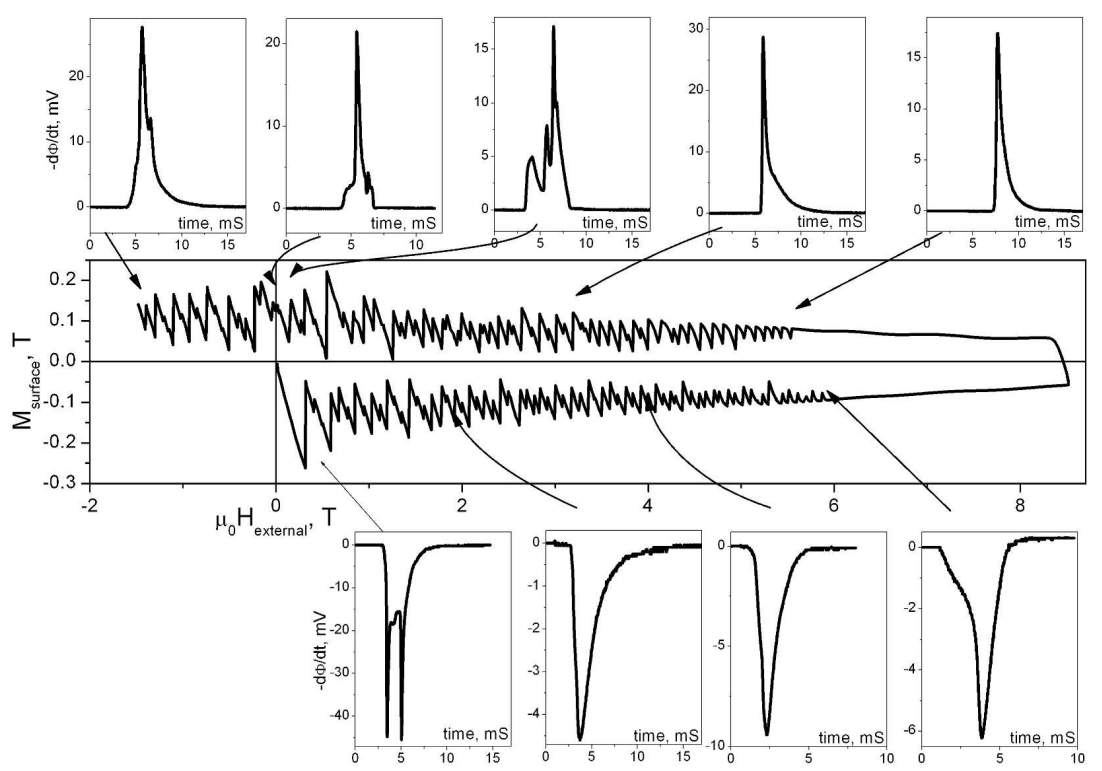

Fig. 4. Surface magnetization hysteresis loop (middle part) and the signals taken from the pick-up coil during the following flux jumps (upper and lower parts) at $2 \mathrm{~K}$. Superconducting sample with two copper disks. 


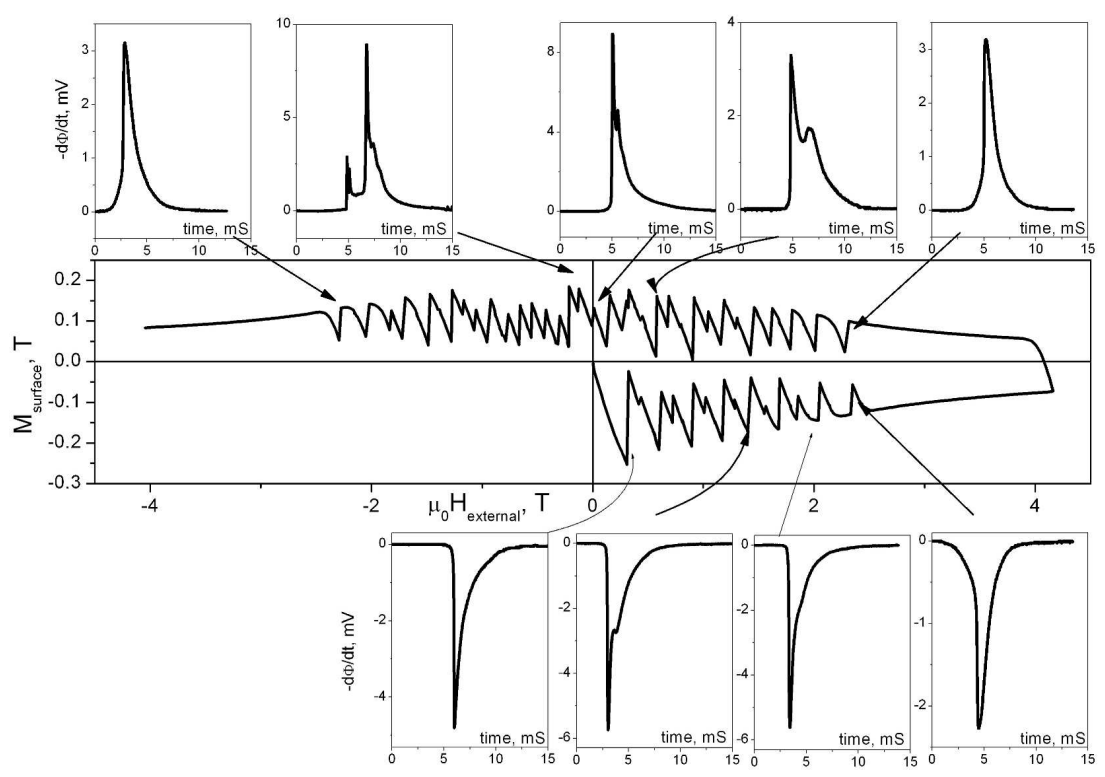

Fig. 5. Surface magnetization hysteresis loop (middle part) and the signals taken from the pick-up coil during the following flux jumps (upper and lower parts) at $4.2 \mathrm{~K}$. Superconducting sample with two copper disks.

when two copper disks were attached to both surfaces of the investigated sample at $2 \mathrm{~K}$ and $4.2 \mathrm{~K}$, respectively. The most complex fine structure of the flux jumps was observed in remagnetization area and also in the small area before it. In this area each signal taken from the pick-up coil during a flux jump consists of several peaks. Such behavior may be caused by the antivortex phase, which increase the instability of nonuniform mixed state $[6,7]$. The amount of the magnetic flux which enters into the sample during thermomagnetic avalanche $(\Delta \Phi)$ was calculated by integration the signal from the pick-up coil.

It has been established that the presence of the cooper discs decreases the amount of the magnetic flux entering into the sample during a flux jump, and simultaneously increases the time of the flux jump duration, Fig. 6 . At $2 \mathrm{~K}$ with increasing external magnetic field a small decrease in the amount of entering magnetic flux was found.

In the situation without copper disks the time of the flux jumps duration is practically independent of temperature, but the value of the magnetic flux entering into the sample during a flux jump increases very fast with increasing temperature. In the situation with attached copper disks with increasing temperature we observed an increase in the time of the flux jumps duration and a decrease in the amount of the magnetic flux entering into the sample during the flux jump (Fig. 6a,b). 

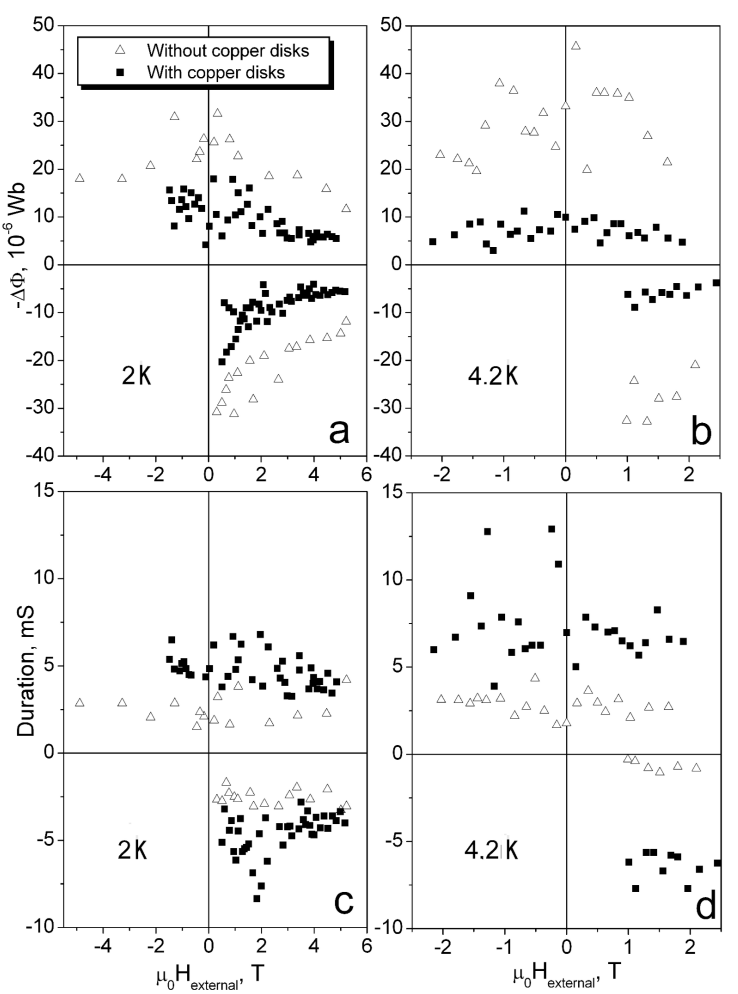

Fig. 6. (a,b) Magnetic field dependence of the amount of the magnetic entering the sample during the following jumps $(\Delta \Phi)$. (c,d) Time of duration of the flux jumps as a function of the external magnetic field. (a,c) The results obtained at $2 \mathrm{~K}$. (b,d) The results obtained at $4.2 \mathrm{~K}$.

To explain qualitatively magnetic field dependence of the amount of magnetic flux entering into the sample during a flux jump we applied the critical state model as well as the Kim-Anderson-like model of the magnetic field dependence of the critical current density $\left(J_{\mathrm{c}}(B)=J_{0} B_{0} / B\right.$, where $B$ - induction of the magnetic field, $J_{\mathrm{c}}$ and $B_{0}$ - constants). Additionally, we assumed that after each jump magnetic field is uniformly distributed in the sample volume and that the following flux jumps are equidistantly distributed along the axis of the external magnetic field. For simplicity we assumed the sample to be in a form of an infinite slab with diameter $2 d$ and the external magnetic field to be parallel to the surface of the slab. In this case the amounts of the magnetic flux which enter into the superconductors volume during the following flux jumps, $\Delta \Phi_{n}$, depend linearly on the jump's number, $n$ :

$$
\Delta \Phi_{n}=2 d L B_{j}\left(1-\frac{\delta_{j} n}{2 d}\right)
$$


where $B_{j}$ - field of the first jump, $\delta_{j}=B_{j} /\left[\mu_{0} J_{\mathrm{c}}\left(B_{j}\right)\right]$ and $L$ - length of the slab measured in the direction perpendicular to the external magnetic field (according to our assumption $L \gg d$ ).

This dependence coincides qualitatively well with our experimental data for the temperature of $2 \mathrm{~K}$ in the situation with copper disks as well as in the situation without copper disks (see Fig. 6a). Unfortunately, at $4.2 \mathrm{~K}$ (Fig. 6b) this coincidence is less visible. To explain the above-mentioned discrepancy as well as to fully understand other phenomena found in our experiment further investigations are necessary.

\section{Conclusions}

Eddy-currents induced in a normal metal being in touch with the superconducting sample moderate significantly dynamics of the flux jumps. The presence of these currents increases the time of the flux jumps duration and simultaneously decreases the amount of magnetic flux, which enters into the superconducting sample during each jump. A simple theoretical model can be applied to describe some of the magnetic field dependences of the amount of magnetic flux entering the superconducting sample during the following jumps.

\section{Acknowledgment}

This work was partly supported by the State Committee for Scientific Research (Poland) under contracts No. 4 T10B 02325 and No. 1 P03B 02027.

\section{References}

[1] S. Field, J. Witt, F. Nori, X. Ling, Phys. Rev. Lett. 74, 1206 (1995).

[2] E.R. Nowak, O.W. Taylor, L. Liu, H.M. Jaeger, T.I. Selinder, Phys. Rev. B 55, 11702 (1997).

[3] K. Behnia, C. Capan, D. Mailly, B. Etienne, Phys. Rev. B 61, R3815 (2000).

[4] R.G. Mints, A.L. Rakhmanov, Rev. Mod. Phys. 53, 551 (1981).

[5] V.V. Chabanenko, V.F. Rusakov, S. Vasiliev, H. Szymczak, A. Nabialek, R. Szymczak, R. Puzniak, J. Jun, J. Karpinski, V. Finkel, L. Huanqian, J. Low Temp. Phys. 130, 175 (2003).

[6] V.K. Vlasko-Vlasov, U. Welp, G.W. Crabtree, D. Gunter, V. Kabanov, V.I. Nikitenko, Phys. Rev. B 56, 5622 (1997).

[7] V.K. Vlasko-Vlasov, U. Welp, G. W. Crabtree, D. Gunter, V. Kabanov, V.I. Nikitenko, L.M. Paulius, Phys. Rev. B 58, 3446 (1998). 